\title{
NUEVAS FORMAS DE NARRAR LA ESCUELA: UNA CONSTRUCCIÓN DE SENTIDOS ALREDEDOR DE LOS DERECHOS DE LOS NINNOS Y LAS NINNAS
}

\section{NEWWAYS OF NARRATING SCHOOL:ACONSTRUCTIONOF MEANINGS AROUND THE RIGHTS OF BOYS AND GIRLS}

\section{NOVAS FORMAS DE NARRAR A ESCOLA: UMA CONSTRUÇ̃̃O DE SENTIDOS AO REDOR DOS DIREITOS DOS MENINOS E AS MENINAS}

\section{Páginas Adriana Castro Camelo \\ 60-72 teachernanita@gmail.com}

Recibido

10 de marzo de 2016

Aceptado 30 de abril de 2016
Magíster en Comunicación y Educación de la Universidad Distrital Francisco José de Caldas. Licenciada en Lenguas Modernas de la Universidad Distrital Francisco José de Caldas. Asesora de proyectos de grado en la Especialización en Comunicación Educativa de la Corporación Universitaria Minuto de Dios - UNIMINUTO. Docente de Lengua extranjera y Ciencias Sociales.

\section{Claudia Gineth Melo Arjona}

claudia.marjona@gmail.com

Magister en Comunicación y Educación de la Universidad Distrital Francisco José de Caldas. Licenciada en educación básica con énfasis en Ciencias Sociales de la Universidad Distrital Francisco José de Caldas. Docente de Ciencias Sociales. 


\section{Resumen}

Este artículo pretende dar cuenta de la dimensión subjetiva e intersubjetiva de los aprendizajes tecnomediados en un tema fundamental como lo es la comprensión y vivencia de los derechos de los niños y las niñas de cuarto grado de dos colegios privados, el cual se deriva del trabajo de investigación Los derechos de los niños y las niñas: construcción de sentidos a través de las narraciones digitales. Tal propósito se realiza en aras de una transformación social de la educación primaria pues allí la instrumentalización de los aprendizajes, pese a la mediación de tecnologías, continúan situando el conocimiento como alejado de la experiencia propia.

\section{Palabras clave}

Construcción de sentidos, derechos de los niños y las niñas, narraciones digitales, percepción, subjetividad 


\section{Abstract}

This article gives account of the subjective and intersubjective dimension of technomedia learning in such a fundamental context as the comprehension and performance of four graders at two private schools. This article derives from Children's Rights: Sense Generation through Digital Narratives. Such a research is performed in order to generate a social transformation in primary education, because learning instrumentalization, in spite of technology use, continues to placing knowledge far from actual experience.

\section{Key words}

Sense Generation, Children's Rights, Digital Narratives, Perception, Subjectivity

\section{Resumo}

Este artigo pretende dar conta da dimensão subjetiva e intersubjetiva das aprendizagens tecno mediados num tema fundamental como é o entendimento e vivência dos direitos dos meninos e as meninas de quarto grau de dois colégios privados, o qual se deriva do trabalho de investigação Os direitos dos meninos e as meninas: construção de sentidos através das narrações digitais. Tal propósito realiza-se em ara de uma transformação social da educação primária pois ali a instrumentação das aprendizagens, pese à mediação das tecnologias, continuam situando o conhecimento como afastado da experiência própria.

\section{Palavras-chave}

Construção de sentidos, direitos dos meninos e as meninas, narrações digitais, percepção, subjetividade 


\section{Introducción}

La emergencia de los saberes que circulan en entornos distintos a la escuela ha generado crisis en la institución en tanto los saberes se han descentrado.

M. Barbero (1991)

Con las transformaciones en las dinámicas sociales y culturales determinadas por la implementación de los medios digitales en todas las esferas de la sociedad, se identifican nuevas formas de construir sentidos determinadas por la inmersión del sujeto en la cultura digital y por el uso y apropiación de las tecnologías. Es de ese modo como, al reflexionar sobre las transformaciones que el sujeto y la sociedad han derivado de su inserción en lo digital, se hace necesario replantear los procesos formativos, los cuales necesitan de la inclusión de nuevas formas de educación que se adapten a los requerimientos de la sociedad y los individuos a los que se educa; esto significa que las Tecnologías de la Información y la Comunicación - Tic- han determinado diferentes maneras de apropiación de conocimiento, las cuales requieren de la implementación de herramientas que atraigan el interés de los estudiantes y permitan una mayor interacción además de la comprensión del entorno digital.

El presente artículo es producto de una investigación realizada en la escuela, gestada en nuestra práctica diaria - la docencia - como resultado de la inquietud por conocer las diferentes maneras en que los niños y niñas de cuarto grado construyen sentidos en torno a una temática que les inquieta y que tiene que ver con los fenómenos sociales que presencian en su cotidianidad: los derechos de las niñas y los niños. También se pretende la búsqueda de alternativas que motiven y faciliten el aprendizaje a través de la incorporación de Tecnologías de la Información y la Comunicación.

Así, bajo el anterior postulado surgió el proyecto "Los derechos de las niñas y los niños: construcción de sentidos a través de las narraciones digitales”, el cual busca dar cuenta de la manera en que las narraciones digitales producidas por los estudiantes constituyen un vehículo para el reconocimiento de sus dinámicas de vida, sus percepciones sobre sus derechos, la vulneración de los mismos, la manera en que se hacen valer, su visión de mundo, parte de su cultura y de su forma de percibir su entorno, por medio del desarrollo de habilidades gestadas en el trabajo colaborativo desde los entornos digitales, todo ello con el fin de identificar la forma en que construyen y reconstruyen sus propios sentidos.

Para tal objetivo se desarrolló una unidad didáctica que soportó la realización de narraciones digitales por parte de estudiantes de cuarto grado de dos colegios privados, a saber, el Colegio Mayor de Los Andes y el Gimnasio 
Moderno Summerhill —quienes interactuaron vía Skype-, narraciones que permitieron conocer las diferentes percepciones de los niños sobre el tema, así como identificar el valor de las narraciones digitales como foco del trabajo colaborativo e interactivo y su papel en la construcción y deconstrucción de subjetividades.

\section{¿Por qué preguntarse sobre la construcción de sentidos en el marco de las tecnomediaciones?}

\section{En el centro de la transformación educativa están las personas, no la tecnología.}

N. Segovia (2010)

En la actualidad, las diversas fuentes de aprendizaje centran su origen en la internet, las redes sociales digitales, las Tecnologías de la Información y la Comunicación, entre otras, con una amplia oferta de contenidos para los niños y niñas que se asemejan a los de los currículos escolares; no obstante, éstos se ofrecen en entornos que no necesariamente tienen finalidades educativas, sino de entretenimiento o noticiosas, aunque de igual manera ofrecen oportunidades de aprendizaje.

Por lo anterior, se puede decir que esta situación ha dado lugar a una serie de cambios en la escuela, los cuales van desde la inclusión de las tic en los contenidos curriculares - bajo el fin único de tener una escuela actualizada en tecnología - hasta procesos menos instrumentales que entienden que los cambios tecnológicos incluyen cambios en las subjetividades; es decir, en el último caso se exploran didácticas tecnomediadas con el objetivo de potenciar no sólo los aprendizajes que se trazan en un diseño curricular, sino también las formas de aprehender críticamente el mundo desde los contextos en que los niños y niñas experimentan sus vidas.

Es por esto que al emprender este proyecto se partió de un lugar común tanto en las prácticas diarias como en las investigaciones del campo de la comunicación-educación: el uso de narraciones digitales como herramienta de construcción de sentidos en un contenido específico, los derechos de las niñas y los niños. Los niños y las niñas entre los nueve y diez años poseen una gran capacidad de indagación y una curiosidad innata por comprender cómo funciona el mundo, quiénes somos, cómo nos expresamos, cómo nos organizamos, etcétera. De igual manera, tienen un marcado carácter colaborativo de aprendizaje para resolver indagaciones que surgen alrededor de cualquier temática. La enseñanza sobre los derechos de los niños y las niñas llama la atención entre ellos dado que los motiva a indagar sobre sus perspectivas personales y colectivas por medio de la interacción con sus pares.

En el marco de la enseñanza de los derechos, el Ministerio de Educación Nacional (2012) establece la enseñanza de los derechos de los niños y las niñas como parte formal del currículo escolar para ser impartida en pro de la construcción de sociedades democráticas y pacíficas —respetuosas de la dignidad inherente a cada individuo para ser reconocidos como iguales en medio de las diferencias-y la promoción de la libertad de crear y transformar el mundo para beneficio individual y colectivo.

La idea de llevar a cabo un proyecto que involucrara la interacción entre niños y niñas de cuarto grado de dos colegios, con diferentes enfoques en educación y en su contexto social, permitiría que los estudiantes investigaran y realizaran narraciones digitales que dieran cuenta de la forma en que construyen sentidos alrededor del tema de sus derechos. A partir de ello, se realizó la secuenciación de contenidos y se creó una unidad didáctica que serviría de apoyo para que los dos grupos de los dos colegios pudieran compartir experiencias respecto a sus propias realidades. 


\section{Hacia el desarrollo de una perspectiva teórica}

Los sentidos tienen por objeto la construcción de un mundo que se asemeje a las experiencias reales.

E. López (2008)

Hablar de construcción de sentidos nos remite a hablar del ser humano, en su creación de múltiples posibilidades como sujeto, según su desarrollo en el proceso de socialización. Dicho proceso logrará su conformación como persona y, consiguientemente, dará sentido a su existencia (Castro \& Melo, 2016). Al construir sentidos, las acciones realizadas por el sujeto llevan un «significado individual» de lo experimentado, además de un significado social "como proceso que requiere del papel mutuo de los participantes en la construcción del significado en cuanto a la naturaleza social» (Vizer, 2006). La interacción permite la unidad y la diversidad cuando se tienen en cuenta los significados compartidos, los sujetos independientes, pensantes, con procesos de vida distintos que se dan en contextos específicos o frente a situaciones concretas. Esto es a lo que González-Rey (2011) denomina subjetividad social, una categoría que reconoce que los sentidos y significaciones que las personas atribuimos los construimos en un proceso intersubjetivo.

De igual manera, Vizer (2006) afirma que:

Comprender la construcción de sentidos en niños y niñas dentro de un significado social es importante en términos de comprender la forma en que construyen su mundo y enfrentan sus experiencias desde que se reconocen a sí mismos hasta formar parte del entorno social. (p. 17).

Tal proceso se presenta en los sujetos durante toda la vida y está expuesto a cambios sociales ya que en el período de la infancia se vive una forma de construcción social de sentidos que puede variar en la edad adulta de acuerdo al entorno en el que se desenvuelve.

De lo anterior se puede colegir que la construcción del sujeto nace en el momento en que el niño es reconocido a sí mismo como persona social con derechos y deberes; asimismo, el niño es un ser capaz de reconocer su dignidad dentro de un contexto que le posibilita construir sus propios sentidos con respecto a sus derechos durante su infancia.

En referencia a esto, es necesario realizar una distinción entre los términos infancia y niño, pues el primero está relacionado con las condiciones socialmente construidas que se atribuyen a los niños en diferentes contextos históricos, sociales y culturales (Usaid, 2014), en tanto que el segundo alude a:

[...] todo ser humano menor de dieciocho años de edad, salvo que, en virtud de la ley que le sea aplicable, haya alcanzado antes la mayoría de edad, el cual, por su falta de madurez física y mental, necesita protección y cuidado especiales, incluso la debida protección legal, tanto antes como después del nacimiento. (Unicef, 2006, p. 9).

La infancia constituye un espacio de tiempo en el que se deben dar los aprendizajes que cooperen tanto con la construcción de sujeto como con el desenvolvimiento en la sociedad. Gaitán (2006) nos indica en relación a esto que «la infancia se experimenta como una realidad objetiva, que se internaliza mediante la socialización, la cual indica cómo deben comportarse los adultos con los niños y lo que deben hacer éstos para llegar a ser adultos» (p. 68). A partir de esta premisa surge el cuestionamiento sobre el papel de los niños en el conocimiento y el ejercicio de sus derechos para rescatar de cierta manera la titularidad activa en esto, ante lo cual es menester acudir a los procesos pedagógicos en el aula para dotarlos del conocimiento de sus derechos, de su autonomía y, de esa forma, darles 
su lugar como personas dignas de aplicárseles derechos y de responder a deberes (Kant, como se citó en Galvis, 2006).

Alrededor del tema de la infancia es pertinente tener en cuenta que las condiciones del entorno social determinan los procesos de socialización, la vivencia de sus derechos, los núcleos de construcción de subjetividades y la influencia de factores como el consumo y los medios. Es por esto que en el contexto escolar — donde el niño desarrolla gran parte de su subjetividad- surgen nuevas disciplinas que permiten interactuar mientras se aprende. Ahora bien, hay que reconocer que las tramas culturales de la humanidad han cambiado, y una institución como la escuela, que tiene entre sus fines más visibles la formación de sujetos, es afectada por estas nuevas maneras de comunicarse y de interactuar. Lo fundamental aquí es reconocer la importancia del uso adecuado de las Tecnologías de la Información y la Comunicación.

Las narraciones digitales son un claro ejemplo de ese tipo de inclusiones mediatizadas de la escuela ya que ellas reconocen todas las características y el potencial en cuanto a interacción y aprendizaje colaborativo se refiere. Dichas características son: 1) descripción de historias con temas cotidianos usando tecnología (Ohler, 2006); 2) la hipertextualidad, en la que, como nos indica Jaime Alejandro Rodríguez (como se citó en Arrieta, 2001, p. 39), «el autor no se impone como figura de autoridad, confía en su lector y le otorga autonomía para que actúe también creativamente, según su interés inmediato, eligiendo, creando o extendiendo los trayectos disponibles»; y 3) el uso de diferentes técnicas narrativas que en las formas de narración anteriores se habían visto limitadas, tales como el texto, la voz, la imagen, etc. (Bolz, 2007).

Las características que tienen estas herramientas desarrolladas dentro del campo de la comunicación digital interactiva favorecen, según Scolari (2008), la creación de nuevos entornos, de nuevas concepciones espaciotemporales y de nuevas identidades construidas a partir de esa interacción con espacios virtuales dado que, además de tener un contenido temático y conceptual, desarrollan nuevas formas de percepción, sensibilización y relación con el medio, lo cual propicia la formación de personalidades democráticas y la construcción y reconstrucción de sentidos.

\section{Construyendo sentidos alrededor de los derechos. La metodología de esta experiencia}

¿Por qué en ese video todos los niños pobres son negros y los ricos son blancos? ¿Acaso los niños blancos no pueden ser pobres? Luisa Bacca, Colegio Mayor de Los Andes

\section{El diseño pedagógico}

Durante el desarrollo de la unidad didáctica y de las clases de ciencias sociales en los dos colegios se pudo apreciar la motivación de los estudiantes por comprender el porqué de las distintas dinámicas que se dan en su contexto, específicamente, en relación al trato y la igualdad de las condiciones de los niños y las niñas. Esto atiende al cuestionamiento sobre las distintas percepciones, sentimientos y posturas que consolidan la subjetividad de los estudiantes en torno a una temática específica, la cual se relaciona con su diario vivir en los diferentes contextos en los que interactúan, y la manera en que dicha visión de mundo se discute y reflexiona a partir de una intersubjetividad dada gracias a la interacción y que desencadena la consolidación de nuevos sentidos del mundo.

Lo anterior, ligado a la importancia de las competencias ciudadanas en la enseñanza escolar y la construcción de ciudadanía y reconocimiento de los derechos, se convierte en una indagación fundamental para conocer la manera en que la vida escolar y las prácticas gestadas en ella construyen y reconstruyen sentidos en los niños y las niñas, permitiendo su empoderamiento con base en el aprendizaje y conocimiento de sus derechos, al 
comprenderse como sujetos políticos y como parte de una sociedad.

Los niños y las niñas tuvieron una sesión extra antes de comenzar a indagar sobre sus derechos. Esta sesión sirvió para que se conocieran vía Skype, se hicieran preguntas acerca de ellos mismos, tuvieran la oportunidad de conocer sus colegios, preguntar por sus formas y estilos de vida, entre otras actividades. Luego de conocerse, los y las estudiantes de los dos colegios prepararon las preguntas con las cuales darían comienzo a sus indagaciones sobre sus derechos. Teniendo en cuenta las preguntas que surgieron, las docentes procedimos a realizar la unidad didáctica y a planear la ruta que llevaría a los y las estudiantes a investigar, comprender, conocer y expresarse acerca de sus derechos, y a nosotras a comprender cómo ellos y ellas construyen sentidos alrededor de este tema.

En la primera sesión, los niños y las niñas de cada colegio observaron los videos sugeridos por las docentes y se organizaron en grupos de discusión para comentar algunos casos de vulneración de derechos que ellos conocían. Acto seguido, se encontraron a través de Skype y hablaron de lo discutido. Para la segunda sesión, los niños y niñas prepararon una pequeña presentación sobre la pregunta que guiaría esta reunión; a partir de ello, se realizó la lectura de las historias de vida que ellos conocen y comenzó la elaboración de los primeros borradores de las historietas. Una vez realizadas las secuencias gráficas, se elaboró el guion y el audio para recrear un video en el que se explicaron dichos casos. La digitalización de las imágenes estuvo apoyada por las docentes.

En la tercera sesión, el eje central se trataba sobre quiénes se encargan de cuidar los derechos de los niños y las niñas; para esto se organizaron los y las estudiantes de los dos colegios para conversar de los conocimientos previos de los agentes que protegen los derechos de los niños y las niñas. Posteriormente se realizaron las historietas acerca del tema central: elaboraron un guion, determinaron quién representaría a cada uno de los personajes, y le dieron vida a la narración que fue grabada por los mismos estudiantes.

La siguiente sesión estuvo dividida en dos clases en las que se elaboraron las narraciones digitales con las herramientas Powtoon, Goanimate, Pixton, Toondoo, Makebeliefcomix y Storybird, y se mostró el producto final de toda su investigación. Finalmente, se socializó el trabajo realizado también mediante Skype. Allí, los niños y niñas discutieron acerca de los videos de sus compañeros, lo que nos dio paso a la elaboración de las conversaciones guiadas que nos servirían de herramienta para la recolección de más datos.

\section{El análisis de los datos}

Esta investigación se enmarca dentro del paradigma cualitativo que permite comprender las experiencias que hacen posible la reflexión de vivencias en términos de permanencias y transformaciones de cada individuo, teniendo en cuenta los factores particulares del contexto, las relaciones sociales, el proceso vital, entre otros aspectos. Con base en ello, se da una reflexión sobre nuestras acciones cotidianas y se busca un cambio, por lo cual se adopta como método de investigación la Investigación Acción en Educación -iae-. Las técnicas de recolección de datos empleadas consistieron en el análisis narrativo, que implica la extracción de información de las narraciones digitales construidas por los niños y niñas, y las conversaciones guiadas, que posibilitan una discusión menos estricta y esquemática que les permite a ellos expresarse y a nosotras, como investigadoras, conocer sus opiniones, saberes, sentimientos y formas de ver el mundo a partir de lo que dicen y conforme se van desarrollando los temas. Esto nos ayuda a conocer su realidad social y a acceder a sus significados y sentidos sobre sus derechos, para luego analizarlos en su relación con los procesos de construcción de los mismos.

El proceso de análisis de datos se realizó siguiendo la ruta de la teoría fundamentada, la cual permitió 
el desarrollo y la construcción teórica, en el contexto de la investigación, desde el análisis de los datos y su conceptuación; de esta manera, se establecieron unas categorías iniciales a partir de los elementos esenciales de nuestra pregunta de investigación. Luego se extrajeron sus propiedades y dimensiones con el fin de buscar conexiones y relaciones de igualdad o diferencia y, así, establecer los criterios base con los cuales se inicia el análisis de datos, los cuales fueron alimentándose y reformulándose en la medida en que los datos se iban analizando. Posteriormente, se codificó la información obtenida de narraciones y conversaciones guiadas, se agrupó, clarificó y sintetizó en matrices de análisis, las cuales posibilitaron la triangulación de la información y sus comparaciones para lograr el análisis. De ese modo surgieron nuevos criterios de análisis contextual y se vincularon la teoría y la práctica para analizar de manera abstracta la realidad encontrada, buscar conexiones y generar una teoría más cercana a esta realidad.

\section{Los procesos de socialización, el conocimiento de los derechos y el de- sarrollo del sujeto: los resultados de esta investigación}

Todo niño viene al mundo con cierto sentido del amor, pero depende de los padres, de los amigos, de su entorno que este amor salve o condene. C. Anderson (2007)

A través del análisis de las narraciones digitales, y teniendo en cuenta sus procesos de socialización, el conocimiento o desconocimiento de sus derechos y el desarrollo de ellos y ellas como sujetos y parte de una sociedad, pudimos observar que los niños y niñas de cuarto grado construyen sentidos alrededor de sus derechos.

Uno de los factores decisivos en el desarrollo del niño es el proceso de adaptación e incorporación a la cultura, el cual está establecido por el entorno en el que desarrolla como ser en sociedad y que, a su vez, está determinado por la familia, en la que se dan los primeros procesos de socialización. En la familia se tejen los primeros lazos de afecto, así como se dan las primeras relaciones de construcción de sentidos por medio del lenguaje, la interacción y las significaciones que se gestan a partir de dicha interacción. Para Palacios (como se citó en Muñoz Silva, 2006):

[...] la familia es el contexto más deseable de crianza y educación de niños, niñas y adolescentes, ya que es quien mejor puede promover su desarrollo personal, social e intelectual y, además, el que habitualmente puede protegerlos mejor de diversas situaciones de riesgo. (p. 148).

Y, en efecto, esto es lo que debería garantizar tal institución: el desarrollo y la protección del menor. Así, en el proceso de construcción de sentidos que hacen los niños y niñas, la familia es la principal responsable, dado que a través de ella se percibe un modo de vida determinado que se ve expresado en actividades y conductas específicas, las cuales conformarán las subjetividades del niño o la niña. Ello se debe a que es allí donde se encuentran los modelos de aprendizaje necesarios para el desarrollo de las habilidades que les servirán para afrontar las diversas situaciones que brinda el entorno social.

De otro lado, los derechos de los niños se enmarcan dentro de la universalidad, por ende, deben ser adaptados a todos los niños y niñas en el mundo. Sin embargo, en una sociedad poco equitativa, vemos cómo las posibilidades no son las mismas para todos puesto que los niños y niñas, además de la cultura, adquieren las condiciones socioeconómicas de la familia en la que nacen y crecen. Por lo anterior, es importante entender que la construcción de sentidos en los niños y niñas se centra en un significado social relevante en términos de la comprensión de la forma en que ven su mundo y enfrentan sus experiencias desde que se reconocen 
a sí mismos hasta formar parte del entorno social; para esto, es menester reconocer sus condiciones de vida basadas en sus propias subjetividades.

Otra forma de construir sentidos está dada por el papel de los medios y la influencia que éstos ejercen en sus subjetividades; para ellos y ellas, los medios no sólo constituyen su principal fuente de información sino también su más importante forma de entretenimiento. No es un secreto que la televisión influye en el conocimiento que los niños y las niñas tienen sobre sus derechos, ya que la continua observación de escenas violentas efectivamente genera la repetición de ciertas ideas en ellos y ellas, lo que causa que se modifique y afecte la manera en que los niños y las niñas perciben y comprenden la realidad. Según Folegotto y Tambornirno (2005), "los niños comienzan a registrar a partir de los 3 años de edad todo tipo de conductas positivas o negativas a raíz de la influencia de los medios de comunicación» (p. 37).

La interacción que brindan los medios masivos como la internet se gesta tanto en redes sociales como en aplicaciones que sirven para comunicarse. El lenguaje que se establece alrededor de esta interacción permite la construcción colectiva de significados ligados a la cultura y el contexto, por lo cual, siempre en estos procesos, se aceptan la expresión de ideas, de inquietudes y nuevos conocimientos, así como la evaluación, confrontación, transformación y definición de percepciones e ideas. Empero, la interacción de los estudiantes no sólo se da alrededor de la tecnología, sino que está dada entre pares y adultos. Para ellos, la interacción está mediada por quien satisface una necesidad y quien la recibe. A partir de ello, se puede decir que el entorno se convierte en el espacio de encuentro con el otro para construir significados de acuerdo a las interacciones que el ser social tenga en él (Bruner, 1993).

Otro aspecto importante en la construcción de sentidos de los niños y las niñas tiene que ver con los agentes vulneradores de sus derechos y que se encuentran en su contexto cercano. Según los niños y las niñas sujetos de esta investigación, cuando el entorno social es el que condiciona las posibilidades de disfrutar los derechos, no hay vulneración; de acuerdo a ellos, la vulneración de los derechos se da cuando los niños asumen las responsabilidades de los adultos, son maltratados o no deseados. De igual manera, la vulneración ocurre cuando son amenazados, entrenados para la guerra, violados 0 abandonados. Así, la construcción de sentidos se ve relacionada con la concreción de propuestas desde el entorno en donde sus derechos son reales en tanto se cumplan, se respeten, se conozcan, se haga uso de ellos y no se permita su vulneración.

Lo anterior está relacionado con la forma en que los estudiantes perciben y sienten sus derechos. Esto contribuye a su desarrollo como sujetos ya que propende por la conformación de un universo de subjetividades que exigen la configuración de espacios que acerquen las diferencias o excluyan aquellas certezas absolutas, lo que aporta a un proceso de construcción de sentidos individuales en medio de una actividad social.

\section{Los frutos de esta experiencia}

No hay enseñanza sin investigación
ni investigación sin enseñanza.
P. Freire

Al terminar este proyecto aparecen múltiples percepciones sobre el modo en que los niños y las niñas construyen sentidos en el tema de los derechos humanos —DDHH - alrededor de su entorno, el cual está relacionado con la familia, la sociedad y los medios de comunicación. De otra parte, sus construcciones de sentido tienen que ver con las condiciones económicas en que viven, el conocimiento de sus derechos, las percepciones que tienen sobre el papel del gobierno en la sociedad, la construcción de ellos y ellas como sujetos y la comunicación y convivencia con quienes comparten su entorno social. 
Para ellos, la experiencia de interactuar con sus pares de otro colegio fue importante en razón a que conocieron otros puntos de vista de niños y niñas de su edad en entornos diferentes, más aún, superando la distancia y las condiciones en que se dieron. Así pues, en primer lugar, como fruto de esta experiencia, se concluye que la interacción dada entre ellos establece una integración no sólo en cuanto a su ser social sino también en cuanto a sus conocimientos y percepciones sobre sus derechos. El hecho de ver diferentes puntos de vista y formas de vida hizo que los niños y niñas abrieran sus ojos a otras realidades que les permitirían evaluar la forma en que viven sus derechos.

Asimismo, cada creación, en referencia a las narraciones digitales realizadas por los estudiantes, diversificaba su forma de pensamiento y desde el momento mismo de sus prediseños en papel se hacía evidente que la temática de sus derechos propiciaba la integración entre el concepto y la capacidad de entender de forma múltiple lo que tradicionalmente se podría pensar como separado. En este sentido, el pensamiento narrativo con sus conexiones, relaciones y puntos de vista se convirtió en el escenario propicio para poner sobre la arena digital diferentes voces y generar espacios de construcción que permitieran un verdadero escenario comunicativo en el que las verdades fuesen relativas, no se pudiera afirmar una última palabra y se tejieran diversas formas de ver el mundo y construir sentidos.

De igual manera, en lo que respecta a la construcción de un entorno social, se logró hacer evidente que hacerlos parte del mundo de los estudiantes y darles significación desde la creatividad es el mejor camino para constituirlos como un elemento motivador y, así, comprender el modo en que se vive, se percibe y se alimenta lo relacionado con derechos humanos; ello, sin perder de lado la senda que ellos mismos trazan en sus propios contextos, pero agregando el aporte a sus subjetividades, asociadas a las experiencias externas.
En cuanto a las representaciones sociales, la construcción de sentidos se desarrolla merced al conocimiento producido por la interacción entre individuos - la cual orienta sus prácticas cotidianas- y no sólo aparece como una construcción intelectual apoyada en cierto sistema de informaciones, sino que expresa formas simbólico-emocionales que tienen que ver con la configuración subjetiva de quienes viven una determinada experiencia.

Entonces, esta experiencia nos permite ver la intersubjetividad en la construcción del sujeto y los conceptos de interioridad y exterioridad que se desarrollan en torno a la forma en que se relaciona con otros y otras, además de los sentidos y significaciones circundantes en esa relación intersubjetiva para constituir una subjetividad. Los niños y niñas crean sus propias representaciones sociales dentro de la articulación de la subjetividad social y la individual, defendiendo la importancia del sujeto en su carácter generador de los espacios sociales en que actúa y, para su caso particular, en la formación que están desarrollando sobre рDHн.

Para nuestros niños es claro - aunque no directamente, sino a través de sus acciones- que la subjetividad social es la forma en que se integran sentidos y configuraciones de diferentes espacios sociales, lo cual forma un verdadero sistema en el que lo que ocurre en cada espacio social concreto —familia, escuela, grupo informal, etc.— está alimentado por producciones subjetivas de otros espacios sociales.

Ahora bien, en la atmósfera de los medios y las тic, lo importante de esta experiencia se relaciona con el proceso dado por el tipo de narraciones que expresan el carácter emocional de sus autores, quienes dan a conocer sus construcciones de sentido y las llevan a escenarios de pensamiento y cambio de conciencia. El campo de la comunicación-educación plantea que lo importante no son las herramientas que se utilicen en tecnología, sino la forma de entender la cultura por encima de los instru- 
mentos (Riveros, 2014). De esa guisa, cuando los niños y niñas pensaban en la estructura de sus narraciones, evidenciaban entender que el eje central estaba en la manera de sentir la realidad, aunque manejaran un "discurso audiovisual y mediático» que les serviría de herramienta para desarrollar su creatividad y formas de expresarse. Así, se fortaleció la escritura y la oralidad, se pasó de un proceso memorístico y de reproducción de información a uno de creación, que a su vez les dio autonomía, les permitió el reconocimiento de sus talentos propios, hacer uso de su propia voz y conocerse y posicionarse como sujetos frente a los demás.

Finalmente, el desarrollo de las narraciones incorporó elementos reales de las experiencias de las y los estudiantes - ya sea vivencias propias, situaciones conocidas de su entorno, casos conocidos por otras personas o casos representados en los medios de comunicación de los cuales hacen uso-y posibilitó las reflexiones sobre las mismas, lo cual pudo demostrar la forma en que el proceso de socialización permite la construcción de nuevas posibilidades. Igualmente, al conversar con los estudiantes se evidenció el reconocimiento de sus derechos, así como la creación de nuevos códigos - significados compartidos - para la práctica en su contexto inmediato, lo cual está relacionado con la creación de identidad, desarrollada en lo que Hunderwesser (2011) denominaría «la cuarta piel» y que considera la construcción del sujeto mismo como producto de la relación con el entorno.

\section{Referencias}

Arrieta, A. (2011). Narrativa digital: Concepto y práctica. Narratopedia, un caso de estudio. [Tesis de maestría]. Manizales: Universidad de Caldas.

Baumeister, R., \& Vohs, K. D. (2007). Belief perseverance. En C. Anderson, Encyclopedia of Social Psychology. Thousand Oaks, CA: Sage.

Benejam, P. (1999). El conocimiento científico y la didáctica de las ciencias sociales. Un currículum de ciencias sociales para el siglo XXI: qué contenidos y para qué (pp. 15-26). Sevilla: Díada Editores.

Bolz, N. (2007). Comunicación: Cambios de paradigmas ante el siglo XXI. En J. La Ferla, El medio es el diseño audiovisual. Manizales: Universidad de Caldas.

Bruner, J. (1997). La educación, puerta de la cultura. Madrid: Visor.

Delgado, J. (1995). Métodos y técnicas cualitativas de investigación en ciencias sociales. Madrid: Síntesis.

Fanlo Cortés, I. (2011). «Viejos»y «Nuevos» derechos del niño. Un enfoque teórico. Revista de Derecho Privado (20), 105-126.

Follegoto, I., y Tambornino, R. (2005). Las TIC y los nuevos paradigmas para la educación. TICEC'05. I Congreso de Tecnologías de la Información y la Comunicación (TICs) en la Enseñanza de las Ciencias. La Plata.

Gaitán, L. (2006). El bienestar social de la infancia y los derechos de los niños. Política y Sociedad, 43 (1), 63-80.

Galvis, L. (2006). Los niños, las niñas y los adolescentes. Titulares activos de derechos. Bogotá: Ediciones Aurora.

Goetz, J. P., \& LeCompte, M. (1988). Madrid: Morata.

González Rey, F. (2011). Sentidos subjetivos, lenguaje y sujeto: avanzando en una perspectiva postracionalista en psicoterapia. Rivista di psichiatria, 46 (5-6), 310-314.

López, B. (2008). Imagen y Construcción del sujeto: el sentido y el desarrollo tecnológico. Revista del Centro de Investigación, 8 (29). 
Morduchowicz, R. (2003). El sentido de una educación en medios. Revista Iberoamericana de Educación (32), 35-47.

Ohler, J. (25 de noviembre de 2006). El mundo de las narraciones digitales. Recuperado de http://eduteka.icesi.edu.co/articulos/ NarracionesDigitales

Relaf, Aldeas Infantiles SOS Internacional. (2010). Documento de divulgación latinoamericano. Niños, niñas y adolescentes sin cuidado parental en América Latina. Buenos Aires: Relaf, Aldeas Infantiles SOS Internacional. Recuperado de https://www.relaf.org/ Documento.pdf

Scolari, C. (2008). Hipermediaciones. Elementos para una Teoría de la comunicación Digital Interactiva. Barcelona: Gedisa.
Segovia, N. (15 de octubre de 2010). Nieves Segovia: "Se educa a los jóvenes para un mundo que ya no existe». Recuperado de http://www.abc. es/20101015/contraportada/nieves-segoviaeduca-jovenes-20101015.html

Unicef. (2006). Convención sobre los derechos del niño. Madrid: Nuevo Siglo.

Usaid. (2014). Niños, niñas y adolescentes. Bogotá: Usaid.

Vizer, E. (2009). Un modelo tridimensional de la comunicación. Mediatización y la doble faz de la(s) realidad(es) social(es). Mediaciones sociales (5), 3-22. 